ulceration -3 , leukopenia -4 patients. Patients were treated initially with 2 infusions of rituximab $(1000 \mathrm{mg})$ given once every two weeks with premedication (methylprednisolone $(250 \mathrm{mg}$ ) and chloropyramine). Then all patients received maintenance therapy with rituximab $500 \mathrm{mg}$ every 6 months. Duration of treatment and follow-up was at least 3 years. Median follow-up was 45 months (3690). Patients were evaluated, using immunologic, subjective parameters, salivary/ lacrimal function at baseline and in 24 weeks and then every year during the follow-up. Patients were not concomitantly treated with another immunosuppressant agent or glucocorticoids daily.

Results: All patients were women with a mean age at diagnosis of 49 years [29$70]$ and median duration of disease was 6 years (1-20). The average cumulative dose of rituximab was $10000 \mathrm{mg}(7000-12000 \mathrm{mg})$. The median European Sjögren's Syndrome disease activity index (ESSDAI) decreased from 2.5 (2-3) to 0 $(0-1)(p=0.01)$ by the end of the study. The increase in salivation and lacrimation according to Schirmer test and tear film break-up time was insufficient, however, in all patients the size of the salivary glands normalised and the recurrent parotitis stopped. Furthermore, 7 patients with severe keratoconjunctivitis sicca improved the course of the disease. The healing of corneal ulceration in all patients was observed one year after the initiation of therapy. A significant decrease in the RF level (from $198 \mathrm{IU} / \mathrm{ml}(51-442)$ to $\left.71 \mathrm{IU} / \mathrm{ml},{ }^{30-73} \mathrm{p}=0,002\right)$ and gammaglobulins (from $28 \% \pm 5 \%$ to $19,3 \% \pm 3,5 \%, p=0,001$ ) was observed one year after this study beginning. Unreliable decrease of immunoglobulins was noted in $23 \%$ of patients by the end of the follow-up, that did not increase the risk of secondary infections. We observed good tolerability of therapy, only 4 patients had mild infusion reactions.

Conclusions: Rituximab therapy is highly effective, well tolerated and helps to avoid long-term use of glucocorticoids/cytotoxic agents in pSS.

Disclosure of Interest: None declared

DOI: 10.1136/annrheumdis-2018-eular.5718

\section{AB0505 EARLY AND SUSTAINED REMISSION IN SLE CAN BE ACHIEVED BY USING PULSE METHYL-PREDNISOLONE HIDROXYCHLOROQUINE AND LOW-MEDIUM DOSE- PREDNISONE}

B.R. Ruiz Estévez ${ }^{1}$, G.R. Irastorza ${ }^{1}$, E. Lázaro ${ }^{2}$, I. Ruiz Arruza ${ }^{3}$, P. Duffau ${ }^{4}$, P. Blanco ${ }^{4}$, on behalf of Systemic Autoimmune Diseases Unit of Cruces Hospital. ${ }^{1}$ Systemic Autoimmune Diseases Unit, Cruces Hospital, Bilbao; ${ }^{2}$ Internal Medicine, Bordeaux Hospital, Bordeaux; ${ }^{3}$ Autoimmunity Diseases Unit, Cruces Hospital, Bilbao, Spain; ${ }^{4}$ Internal Medicine, Bordeaux Hospital, Bordeaux, France

Objectives: To compare the different therapeutic schemes used in two longitudinal cohorts of patients with Systemic Lupus Erythematosus (SLE): the Systemic Autoimmune Diseases Unit of Cruces University Hospital, Barakaldo, Spain (cohort C), using a low-medium dose prednisone scheme; and the Internal Medicine Department of Bordeaux, France (cohort B) which follows the standard treatment guidelines.

Methods: We included patients from both cohorts diagnosed with (SLE from year 2000), with a follow-up from the time of diagnosis (inception cohorts) and for at least 5 years. We analysed the different use of glucocorticoids (prednisone and pulse methylprednisolone), hydroxychloroquine (HCQ) and immunosuppressive drugs in both cohorts. To compare the efficacy of both therapeutic schemes, we analysed the rate of clinical remission and clinical remission on treatment, according to the DORIS definitions, during the 5 years of follow-up

Results: 173 patients were studied: Bordeaux (B): 81 and Cruces (C): 92. Baseline clinical variables were similar between both groups, including demographics, autoimmunity and organ involvement. The baseline SLEDAI was: 8 (B) vs. 7 (C), $\mathrm{p}=0.06$. The proportion of patients in treatment with prednisone was similar from year $1: 79 \%(B)$ vs. $72 \%(C), p=0.269$, to year $5: 63 \%(B)$ vs. $55 \%(C), p=0.315$. However, patients in cohort $B$ received higher doses of prednisone: mean maximum dose at year $1: 38 \mathrm{mg} / \mathrm{d}(\mathrm{B})$ vs. $14 \mathrm{mg} / \mathrm{d}(\mathrm{C}), \mathrm{p}<0.05$; at year $5: 8 \mathrm{mg} / \mathrm{d}(\mathrm{B})$ vs. $3 \mathrm{mg} / \mathrm{d}(\mathrm{C}), \mathrm{p}<0.05$; average dose of prednisone at year $1: 15.8 \mathrm{mg} / \mathrm{d}(B) \mathrm{vs}$. $4.4 \mathrm{mg} / \mathrm{d}(\mathrm{C}), \mathrm{p}<0.05$; at year 5: $6.6 \mathrm{mg} / \mathrm{d}(B)$ vs. $3.2 \mathrm{mg} / \mathrm{d}(\mathrm{C}), \mathrm{p}<0.05$. Methyl-prednisolone pulses were ever used in $26 \%$ (B) vs. $42 \%(C), p<0.05$; the mean total pulses per patient was $3(B)$ vs. $7(C), p<0.05$; however, the mean total dose of methyl-prednisolone per patient was similar $1725 \mathrm{mg}$ (B) vs. $1635 \mathrm{mg}$ (C), $p=0.835$, revealing a lower dose per pulse in patients from cohort $C$. The proportion of patients in treatment with $\mathrm{HCQ}$ was lower in cohort $\mathrm{B}$ at year $1: 80 \%(\mathrm{~B})$ vs. $97 \%(C), p<0.05$ and at year 5: 73\% (B) vs. $99 \%(C), p<0.05$. Methotrexate was used more frequently in cohort $C(p<0.05)$ with no differences in cyclophosphamide, azathioprine and mycophenolate use.

Clinical remission in both cohorts was as follows: year 1: $25 \%$ (B) vs. $40 \%$ (C), $p<0.05$; year 2: $29 \%$ (B) vs. $42 \%$ (C), $p=0.07$; year $3: 37 \%$ (B) vs. $42 \%$ (C), $p=0.54$; year $4: 37 \%$ (B) vs. $48 \%(C), p=0.15$; year $5: 36 \%$ (B) vs. $49 \%(C)$, $\mathrm{p}=0.08$.
Clinical remission on treatment: year 1: $43 \%$ (B) vs. $84 \%(C), p<0.05$; year $2: 70 \%$ (B) vs. $87 \%(C), p<0.05$; year 3: 73\% (B) vs. $88 \%(C), p<0.05$; year $4: 73 \%$ (B) vs. $93 \%(C), p<0.05$; year 5: 73\% (B) vs. $93 \%(C), p<0.05$.

\section{Conclusions:}

- The more frequent use of hydroxychloroquine, pulse methylprednisolone and methotrexate in the Cruces cohort resulted in reduced doses of oral prednisone.

- Patients fron the Cruces cohort achieved clinical remission earlier and in a more sustained way during the follow-up.

Disclosure of Interest: None declared

DOI: 10.1136/annrheumdis-2018-eular.7228

\section{AB0506 HYDROXYCHLOROQUINE HAS NO PROTECTIVE EFFECT ON THE DEVELOPMENT OF SYSTEMIC LUPUS ERYTHEMATOSUS IN 7004 PATIENTS WITH PRIMARY SJÖGREN'S SYNDROME: A NATIONWIDE POPULATION- BASED STUDY}

B.-C. Hsu, K.-T. Tang. Department of Allergy, Immunology, and Rheumatology, Taichung Veterans General Hospital, Taichung, Taiwan, Province of China

Background: Hydroxychloroquine (HCQ) has been proposed to be associated with later onset of systemic lupus erythematosus (SLE) ${ }^{1}$ and is widely used in patients with primary Sjögren's syndrome (pSS) which may evolve to SLE. ${ }^{2}$ We want to explore the potentially protective role of $\mathrm{HCQ}$ in the development of SLE among patients with pSS.

Objectives: This study was conducted to assess whether exposure to HCQ in pSS patients is associated with a reduction in the development of SLE.

Methods: This retrospective cohort used claims data from the National Health Insurance Registry Database (NHIRD) in Taiwan. Patients with incident Sjögren's syndrome (SS) from 2000 to 2010 in the Registry of Catastrophic Illness Database (RCIPD) of the NHIRD, which was certified by two rheumatologists, were identified. The date when SS was diagnosed in the RCIPD was defined as the index date. Those who were diagnosed as having SLE, rheumatoid arthritis, polymyositis, dermatomyositis, or systemic sclerosis in the RCIPD before the index date were excluded. Other exclusion criteria included ${ }^{1}$ patients who were diagnosed as having SLE in the RCIPD within one year after the index date, ${ }^{2}$ patients who withdrew from the NHIRD within one year after the index date, and ${ }^{3}$ patients who used oral, intramuscular, or intravenous corticosteroids, methotrexate, azathioprine, leflunomide, sulfasalazine, cyclosporine, tacrolimus, mycophenolate, mercaptopurine, or cyclophosphamide for more than or equal to 90 days within one year before or after the index date. The included SS patients who used HCQ for more than or equal to 90 days within one year after the index date were eligible to HCQ group. The study endpoint was defined as newly-diagnosed SLE in RCIPD or withdraw from NHIRD during the 14 year follow-up period (January 1st, 2000 to December 31st, 2013).

Results: A total of 7004 pSS patients were identified. The mean follow-up time was 6.9 years in the HCQ group $(n=4282)$ and 7.0 years in the non-HCQ group $(n=2722)$. There were 22 newly-diagnosed SLE $(0.5 \%)$ in the HCQ group and 16 $(0.6 \%)$ in the non-HCQ group. The overall event rate of SLE was 8.78/10,000 person-years in the HCQ group and 9.83/10,000 person-years in the non-HCQ group (adjusted hazard ratio $0.97,95 \%$ confidence interval $0.50-1.88$, in a Cox proportional hazard model).

Conclusions: There is no protective effect of HCQ on the development of SLE in patients with pSS.

\section{REFERENCES:}

[1] James JA, Kim-Howard XR, Bruner BF, et al. Hydroxychloroquine sulfate treatment is associated with later onset of systemic lupus erythematosus. Lupus 2007;16:401-409.

[2] McDonagh JE, Isenberg DA. Development of additional autoimmune diseases in a population of patients with systemic lupus erythematosus. Ann Rheum Dis 2000;59:230-232.

Disclosure of Interest: None declared DOI: 10.1136/annrheumdis-2018-eular.3885 